\title{
Smarter Approaches to Dementia
}

\author{
INTRODUCTION \\ Adalsteinn D. Brown, DPHIL \\ Editor-in-Chief, Healthcare Papers \\ Director, Institute of Health Policy, Management and Evaluation \\ University of Toronto \\ Toronto, $\mathrm{ON}$
}

IN THE LEAD essay of this edition of

Healthcare Papers, Frances Morton-Chang and her colleagues (2016) lay out key elements of a dementia strategy: (1) engage people living with dementia in decisions about their own care; (2) support informal caregivers who take care of loved ones with dementia; and (3) maintain persons living with dementia in their homes and community settings as long as it is appropriate.

The appeal of a dementia strategy is clear. Dementia is arguably one of the greatest threats to the sustainability of our health and social services systems with over half a million Canadians living with Dementia (PLWD) today and nearly one million PLWD expected within 15 years (Alzheimer Society of Canada 2017). There is no cure yet for dementia and although prevention and early detection are possible, they are relatively under-addressed across health systems.

But the problems posed by dementia go well beyond those of healthcare cost and utilization. Because PLWD can depend so heavily on informal (spouses, children, and friends) and formal (physicians, nurses, personal support workers) caregivers, they are often referred to long-term care well before their needs necessitate this level of support. This means - as many papers note in this issue - that we need to change how we approach caregivers in our system, recognize their contribution and find ways to support them. It also means that we have to consider how we plan for systems' capacity to respond to dementia. If we rely on traditional, long-term care heavy models of caring for PLWD we will create a capital investment burden on our health system that crowds out investment in hospitals, let alone other necessary investments in our health system. And it means that simple healthcare system-based responses, regardless of whether they occur in hospitals, long-term care homes or the community are unlikely to be effective. Simply putting more resources into our delivery system will not necessarily reduce the burden on caregivers or stem the rise in dementia itself. The supports needed by PLWD and their caregivers go well beyond the health system as they cope with limitations 
on daily activities and burnout for caregivers, increased financial burden and social isolation related to their disease or their caregiving role.

However, it also means that we need to start to create the data systems and instrumentation that allow us to both better organize and manage the care of PLWD and understand better ways of preventing, reducing the impact, and ultimately curing dementia in all its different forms. Recent work supported by the Organisation for Economic Co-operation and Development (OECD 2015) as well as major investments at leading universities have focused attention on the importance of big data - both broad in terms of the scope of data collected and deep in terms of the clinical detail - to meeting the challenge of dementia. First of all, broad data that covers social services, health services, financial status, and other factors are necessary to create scorecards and support improvement efforts that will allow us to structure and manage the population of PLWD. Equally so, linkage of broad deep data - as in the Swedish Brain Power longitudinal studies or Braincode here in Ontario - that allows us to assemble large amounts of genetic, phenotypic and other clinical data will enable us to better understand the many aspects and types of dementia and develop cures and treatments.

Yet we also need to ensure that both policy and data-based approaches to managing dementia recognize the equity impacts of dementia.

But we will not realize the potential of big data for dementia just by throwing more money into grants and other types of funding, just like we will fail to support PLWD by simply providing more care. Rather, new policies that ensure the use of data to structure and support a system for PLWD like scorecards, audit and feedback, or standards are critical to ensure that busy providers and policy makers pay attention to PLWD. New policies that promote the use and re-use of research data and its sharing across teams of researchers are also necessary to quicken the march of discovery.

Yet we also need to ensure that both policy and data-based approaches to managing dementia recognize the equity impacts of dementia. Caregiving continues to fall heavily on women and seniors. When women (or men) take time away from work to take care of loved ones with dementia, the long-term impacts on their income can be substantial. When care needs increase substantially, the impact on savings and retirement income can also be substantial so that income security is threatened and pensions, once thought to be adequate, can come up short. The National Population Health Study of Neurological Conditions reported that "the combined Canadian health care system costs and out-of-pocket caregiver costs amounted to $\$ 10.4$ billion in 2016. By 2031, this figure is expected to increase to $\$ 16.6$ billion" (Alzheimer Society of Canada 2016). If we do fail to address these equity issues we will find that even our best efforts to improve care for PLWD will exacerbate rather than reduce inequities.

Organizations and individuals focusing on dementia and the needs of PLWD should maintain their advocacy and fundraising efforts but if these are not accompanied by efforts to understand, improve, and even restructure our data, care, and caregiver support systems they will have little impact. The papers in this issue provide a series of options on how we can begin to restructure our systems and the work of the OECD can provide strong guidance on how we need to improve our use of data. 


\section{References}

Anderson, G. and J. Oderkirk. 2015. Dementia

Research and Care: Can Big Data Help? Paris,FR:

OECD Publishing. doi:10.1787/9789264228429-en.

Alzheimer Society of Canada. 2016. Prevalence and Monetary Costs of Dementia in Canada. Retrieved March 8, 2017. <http://www.phac-aspc.gc.ca/ publicat/hpcdp-pspmc/36-10/ar-04-eng.php>.
Alzheimer Society of Canada. 2017. Dementia Numbers in Canada. Retrieved March 8, 2017. <http://www.alzheimer.ca/en/About-dementia/ what-is-dementia/Dementia-numbers >.

Morton-Chang, F., A.P. Williams, W. Berta and A. Laporte. 2016. "Towards a CommunityBased Dementia Care Strategy: How Do We Get There from Here?" Healthcare Papers 16(2): 8-32. doi:10.12927/hcpap.2017.25006.

\section{Subscribe now!}

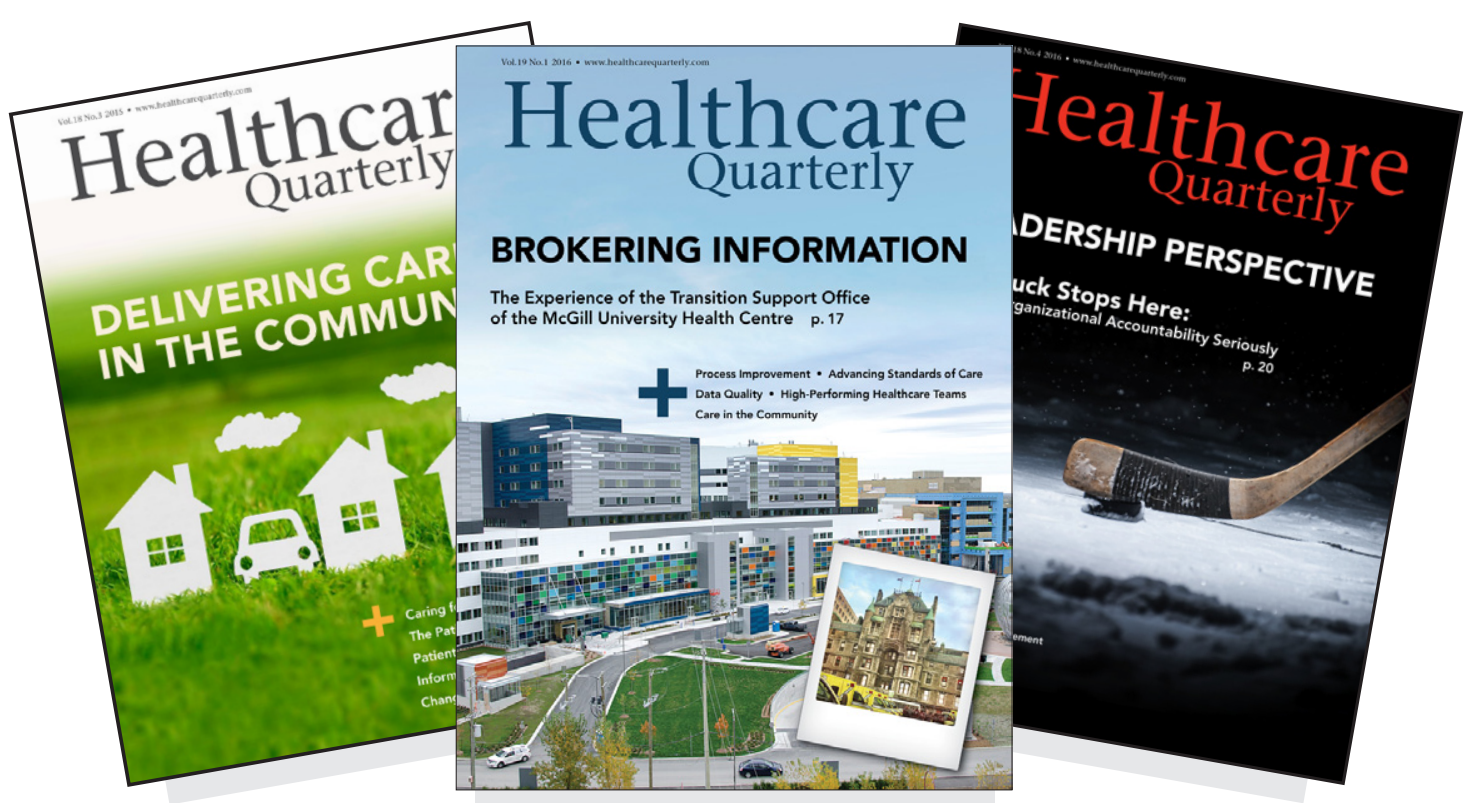

Healthcare Quarterly recognizes, nurtures and champions excellence in the Canadian healthcare system. Its objective is to document and disseminate leading practices in health service delivery and policy development. Excellence is achieved through constant innovation, motivated people and inspired leadership at all levels of the organization. Healthcare Quarterly helps Canadian health system managers anticipate and respond to changing environments, demands and mandates.

\section{HealthcareQuarterly.com}

Longwoods.com 